\title{
Performance of Calorimetry in ALICE
}

\author{
Yuri Kharlov*t‡ \\ NRC "Kurchatov institute" - IHEP, Protvino, Russia \\ E-mail: Yuri.Kharlovecern.ch
}

The ALICE experiment at LHC studies the strong interaction sector of the Standard Model with pp, pA and AA collisions. Within the scope of the physics program, measurements of photons, neutral mesons and jets in ALICE are performed by two electromagnetic calorimeters. Precise and high-granularity photon spectrometer (PHOS) composed of lead-tungstate crystals, along with a wide-aperture lead-scintillator sampling calorimeter (EMCal) provide complementary measurements of photon observables in a wide kinematic range. The calorimeter trigger system allows the experiment to utilize efficiently the full delivered luminosity, recording a data sample enhanced with high-energy photons and jets. Performance of the ALICE calorimeters from proton-proton to heavy-ion collision systems is discussed and illustrated by physics results derived from data collected by ALICE with its electromagnetic calorimeter system.

Sixth Annual Conference on Large Hadron Collider Physics (LHCP2018)

4-9 June 2018

Bologna, Italy

\footnotetext{
* Speaker.

$\dagger$ for ALICE collaboration

$\ddagger$ also in NRC "Kurchatov Institute", Moscow, Russia and MIPT, Dolgoprudny, Russia
} 
The electromagnetic calorimeter system of the ALICE experiment at LHC consists of two detectors: a high-precision photon spectrometer PHOS [1] and a wide-aperture electromagnetic calorimeter EMCal [2]. The choice of the parameters of the calorimeters is driven by the physics objectives which the detectors were designed for. One of the primary goals of the PHOS is studying thermal properties of the hot strongly interacting matter created in heavy-ion collisions, by measuring direct photon radiation at low transverse momenta, $p_{\mathrm{T}}$. This task relies on photon and neutral meson detection in a $p_{\mathrm{T}}$ range from hundreds of $\mathrm{MeV}$ up to a hundred $\mathrm{GeV}$. The EMCal was designed for exploring parton energy loss in the QCD matter via measuring jet spectra, as well as prompt photons and electrons at high $p_{\mathrm{T}}$ up to $250 \mathrm{GeV} / c$. The EMCal detector was installed in ALICE in two stages: the first part, called EMCal, was installed for the LHC Run1, while an extension of this calorimeter, referred to as DCal, was installed for the LHC Run2. In the following both parts are referred to as EMCal, and mention DCal only when the new modules are in question. Basic parameters of the EMCal and PHOS are summarized in Table 1.

\begin{tabular}{|l|l|l|}
\hline & EMCal & PHOS \\
\hline Active element & $\begin{array}{l}77 \text { layers }(1.44 \mathrm{~mm} \mathrm{~Pb}, 1.6 \mathrm{~mm} \\
\text { scintillator) }\end{array}$ & Crystals $\mathrm{PbWO}_{4}$ \\
Molière radius & $3.2 \mathrm{~cm}$ & $2.0 \mathrm{~cm}$ \\
Photodetector & APD $5 \times 5 \mathrm{~mm}^{2}$ & APD $5 \times 5 \mathrm{~mm}^{2}$ \\
Depth & $20 X_{0}$ & $20 X_{0}$ \\
Acceptance & EMCal: $|y|<0.7, \Delta \varphi=107^{\circ}$, & $|y|<0.13, \Delta \varphi=70^{\circ}$ \\
& DCal: $0.22<|y|<0.7, \Delta \varphi=67^{\circ}$ & \\
Granularity & Cell $6 \times 6 \mathrm{~cm}^{2}$, & Cell $2.2 \times 2.2 \mathrm{~cm}^{2}$, \\
& $\Delta \varphi \times \Delta \eta=0.0143 \times 0.0143$ & $\Delta \varphi \times \Delta \eta=0.0048 \times 0.0048$ \\
Modularity & EMCAL: $10+2(1 / 3)$ modules, & $3+1 / 2 \mathrm{modules}, 12544$ cells \\
& DCAL: $6(2 / 3)+2(1 / 3)$ modules, & \\
Dynamic range & $17664 \mathrm{cells}$ & $0-100 \mathrm{GeV}$ \\
Energy resolution $\sigma_{E} / E$ & $0-250 \mathrm{GeV}$ & $4.8 \% / E \oplus 11.3 \% / \sqrt{E} \oplus 1.7 \%$ \\
Distance from interac- & $428 \mathrm{~cm}, 0.7-0.9 X_{0}$ & $460 \mathrm{~cm}, 0.2 X_{0}$ \\
tion point & \multicolumn{2}{|l}{} \\
\hline
\end{tabular}

Table 1: Basic parameters of the ALICE calorimeters EMCal and PHOS.

The ALICE calorimeters record collision data together with other detectors by triggering on minimum-bias beam-beam interactions, but EMCal and PHOS also can serve as self-triggered detectors by recording events characterized by high-energy objects detected in the calorimeters: photons, electrons and jets. These triggering capabilities on rare events with small cross sections allows the experiment to inspect the full luminosity delivered by the LHC. The EMCal and PHOS generate the level-0 (L0) trigger which is fired with a latency of $1.4 \mu \mathrm{s}$ after beam interaction by selecting events with clusters of high energy above a configurable threshold, which is typically set to $2.5 \mathrm{GeV}$ in EMCal and $4 \mathrm{GeV}$ in PHOS. The calorimeter trigger system can also generate the level-1 (L1) triggers with a latency $7 \mu$ s by inspecting events preselected by L0 and searching for events with high-energy photons and jets with more elaborated algorithms. Several L1 triggers 
are generated by the calorimeters. EMCal emits two L1 triggers on photon-like clusters with two energy thresholds which are equal to 4 and $9 \mathrm{GeV}$ in pp collisions, and two triggers on jets with thresholds 16 and $20 \mathrm{GeV}$ in pp collisions. PHOS generates only photon L1 triggers with three thresholds. Performance of the calorimeter triggers can be represented by the ratios $R_{\text {trig }}$ of the photon spectra measured with the corresponding triggers to those from the minimum bias trigger or from the calorimeter trigger with a lower threshold, as shown in the left plot of Fig. 1 for the EMCal trigger. Similar characteristics is the trigger efficiency, the probability of a high-energy photon to generate the trigger, as illustrated on the right plot of Fig. 1 for PHOS. In EMCal, the event rates of L1 triggers with low threshold are too high and are therefore downscaled to accommodate the readout rate accepted by the ALICE data acquisition. The EMCAL L1 triggers with high threshold and the PHOS L0 trigger have rather low event rates and can be recorded without downscaling.
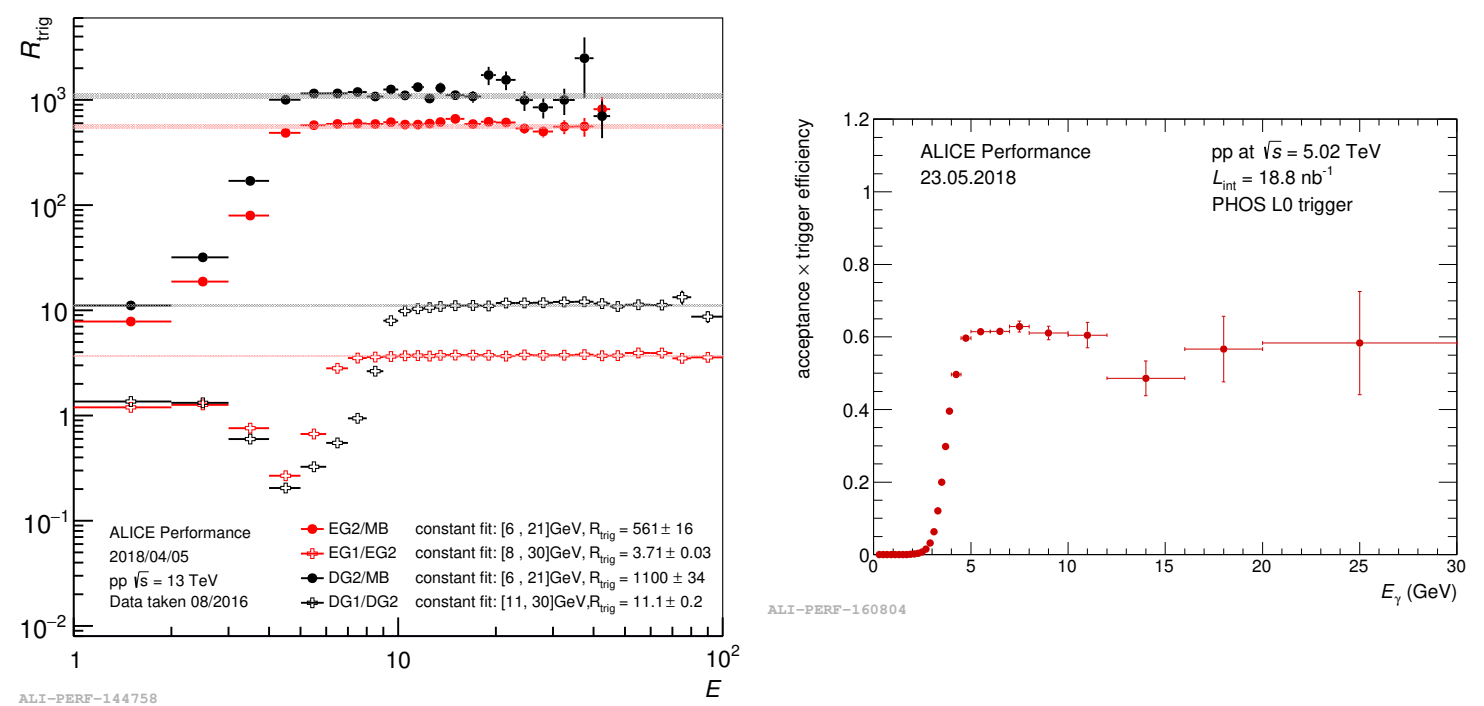

Figure 1: Turn-on curves for EMCal L1 triggers in pp collisions at $\sqrt{s}=13 \mathrm{TeV}$ and for PHOS L0 trigger in pp collisions at $\sqrt{s}=5.02 \mathrm{TeV}$.

The dominant background in the measurement of direct photons is determined by photons from light neutral decays, mainly $\pi^{0}$ and $\eta$, therefore one of the primary tasks for the ALICE calorimeters is a precise measurement of neutral meson spectra in a wide $p_{\mathrm{T}}$ range. Neutral meson spectra measured in pp, pA and AA collisions are important themselves as a sensitive probe to study hard parton energy loss in QCD medium via measurement of the nuclear modification factor $R_{A A}$. The advantage of $\pi^{0}$ and $\eta$ spectra measurement in the ALICE calorimeters with respect to charged hadrons is provided by a much wider energy range accessible for neutral meson identification.

The main method of neutral meson reconstruction in EMCal and PHOS is to build the invariant mass spectra of photon pairs with combinatorial background subtraction. Fig. 2 presents examples of two-cluster invariant mass spectra in various $p_{\mathrm{T}}$ bins in pp collisions at 5 and $13 \mathrm{TeV}$. These spectra show clear peaks corresponding to $\pi^{0}$ and $\eta$ mesons above a combinatorial background. The background has a contribution from correlated cluster pairs coming from the same source, such as jets or secondary interaction of photons with the detector material, as illustrated in Fig. 2. The technique of background modelling by mixed events is deployed in data analysis to subtract the background. The Figure 2 also demonstrates the $\pi^{0}$ and $\eta$ peaks in PHOS at the highest 

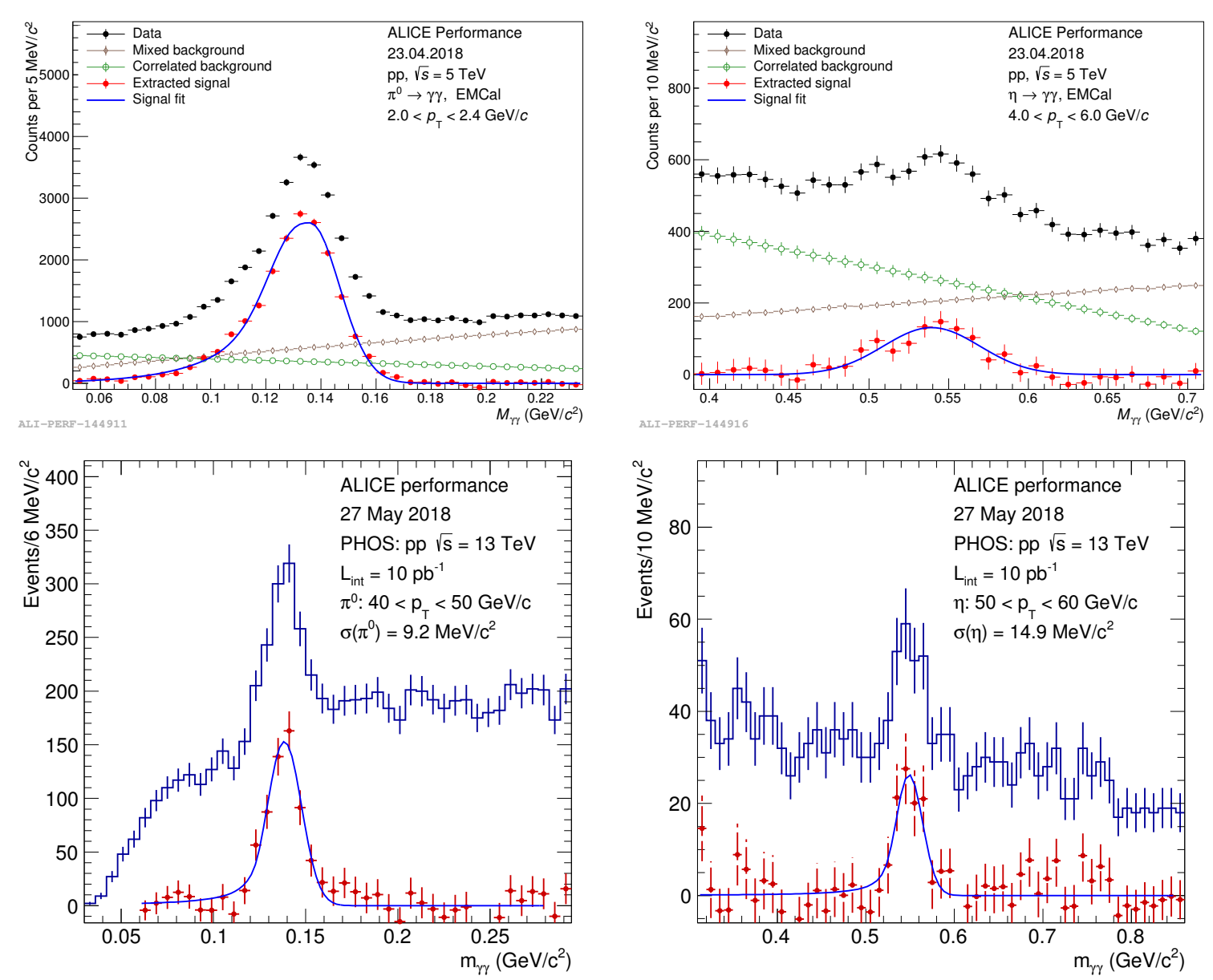

Figure 2: Two-photon invariant mass spectra in pp collisions at $\sqrt{s}=5.02 \mathrm{TeV}$ in EMCal (upper row) and at $\sqrt{s}=13 \mathrm{TeV}$ in PHOS (bottom row) in the $\pi^{0}$ and $\eta$ meson peak regions.

$p_{\mathrm{T}}$ accessible in pp collisions at $\sqrt{s}=13 \mathrm{TeV}$ in 2016-2017 with integrated luminosity $L_{\text {int }}=$ $10 \mathrm{pb}^{-1}$. As one can see, statistics of photon pairs at $40<p_{\mathrm{T}}<50 \mathrm{GeV} / c$ is high enough for the $\pi^{0}$, although $p_{\mathrm{T}}=50 \mathrm{GeV} / c$ is the upper limit of $\pi^{0}$ reconstruction using the invariant mass method because the photons from $\pi^{0}$ decays at higher $p_{\mathrm{T}}$ cannot be separated due to cluster overlap in PHOS. The upper limit of $\eta$ meson reconstruction is driven by statistics only due to a larger opening angle of photons from $\eta$ decay. The ALICE collaboration has performed measurements of $\pi^{0}$ and $\eta$ yields at mid-rapidity in $\mathrm{pp}[3,4,5], \mathrm{p}-\mathrm{Pb}[6]$ and $\mathrm{Pb}-\mathrm{Pb}$ collisions [4] at LHC energies.

Data from the calorimeters, being analyzed together with the ALICE central tracker, allows one to reconstruct electrons matching the calorimeter clusters with tracks propagated to the calorimeter surface. Electrons, unlike other charged particles, deposit their whole energy in electromagnetic calorimeters, and thus the ratio of the calorimeter energy $E$ to the track momentum $p$ is equal to 1. The distribution of $E / p$ ratio of EMCal clusters and matched tracks in $\mathrm{Pb}-\mathrm{Pb}$ collisions at $\sqrt{s_{\mathrm{NN}}}=5.02 \mathrm{TeV}$ is shown on the left plot of Fig. 3. In conjunction with electron identification in the ALICE Time Projection Chamber (TPC), EMCal allows to reconstruct and identify electron in the wide $p_{\mathrm{T}}$ range $p_{\mathrm{T}}<30 \mathrm{GeV} / c$ (Fig. 3, right plot).

In measurements of electrons from semileptonic decays of heavy quarks $c$ and $b$ characterized 

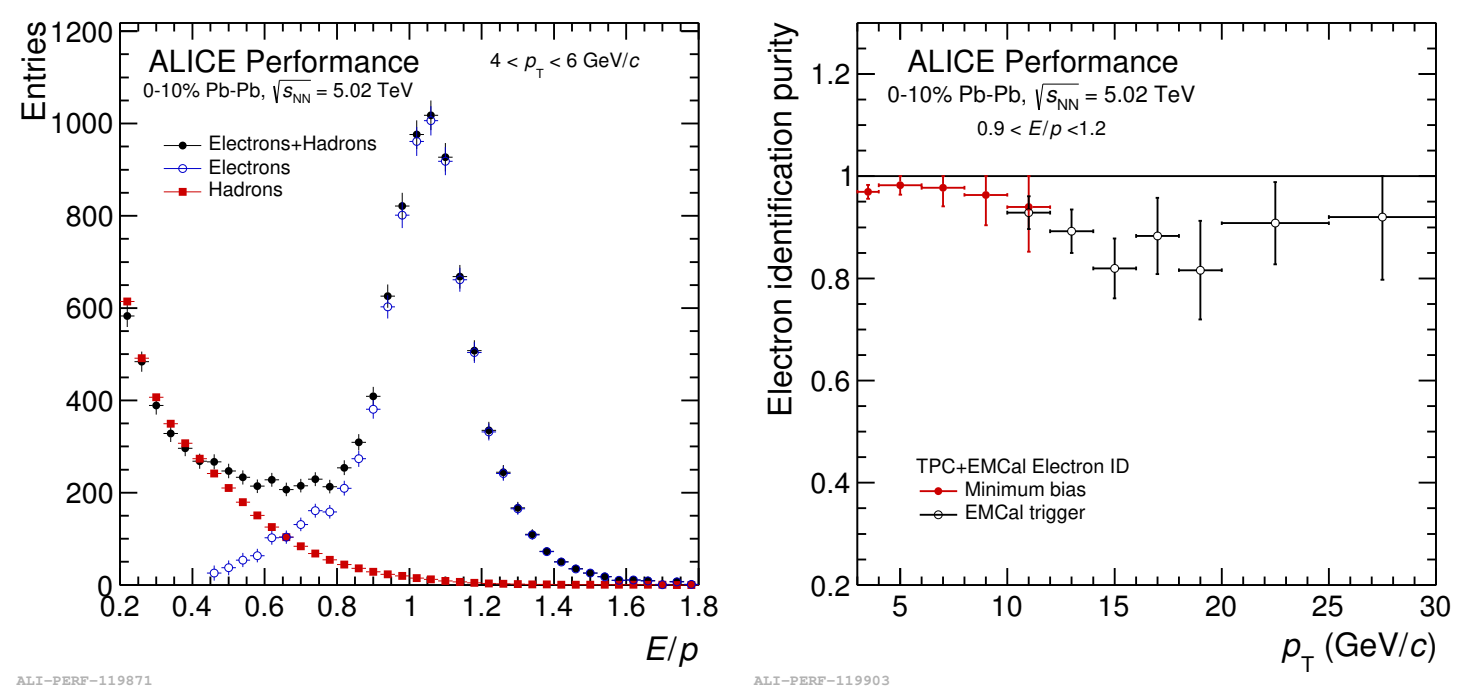

Figure 3: Distribution of electron energy-over-momentum $E / p$ ratio (left) and electron identification purity (right) in central $(0-10 \%) \mathrm{Pb}-\mathrm{Pb}$ collisions at $\sqrt{s_{\mathrm{NN}}}=5.02 \mathrm{TeV}$ in EMCal.

by small cross sections, the EMCal trigger is powerful to select events with high-energy electrons. In $\mathrm{Pb}-\mathrm{Pb}$ collisions at $\sqrt{s_{\mathrm{NN}}}=2.76 \mathrm{TeV}$ the EMCal trigger enhanced electron samples by a factor of 30-40 [7], as shown in the trigger turn-on curves of electron $p_{\mathrm{T}}$ spectra in Fig. 4. Electron identification in EMCal is also used for charmonium reconstruction. Capabilities of $J / \psi$ reconstruction in pp collisions at $\sqrt{s}=13 \mathrm{TeV}$ in ALICE are illustrated by $e^{+} e^{-}$invariant mass spectrum at $11<p_{\mathrm{T}}<30 \mathrm{GeV} / c$ in Fig. 5, where one electron is reconstructed and identified in the TPC and at least one electron with $p_{\mathrm{T}}>7 \mathrm{GeV} / c$ is reconstructed in EMCal and identified by the energy-to-momentum ratio $0.8<E / p<1.3$.

In the jet measurements, the role of EMCal is to trigger events with large energy deposited by photons in the EMCal acceptance, as described in [8], and to reconstruct the neutral jet energy. Charged jet component is reconstructed by the central tracking system. These jet detection capabilities were used in measurements of differential cross section of jets in pp collisions at $\sqrt{s}=2.76 \mathrm{TeV}$ [9], in jet suppression measurements in $\mathrm{Pb}-\mathrm{Pb}$ collisions at $\sqrt{s_{\mathrm{NN}}}=2.76 \mathrm{TeV}$ [10], in dijet correlations in $\mathrm{p}-\mathrm{Pb}$ collisions at $\sqrt{s_{\mathrm{NN}}}=5.02 \mathrm{TeV}$ [8].

To summarize, we conclude that the ALICE electromagnetic calorimeters contribute to the ALICE physics program with photon, neutral meson, electron, jet measurements in pp, pA and AA collisions. The trigger system based on the electromagnetic calorimeters allows ALICE to enhance the collected sample with high- $p_{\mathrm{T}}$ events, and thus utilize the full luminosity delivered by the LHC. Photon, neutral pion and electron identification is elaborated in order to ensure high purity and efficiency.

This work was supported by the RSF grant 17-72-20234. 


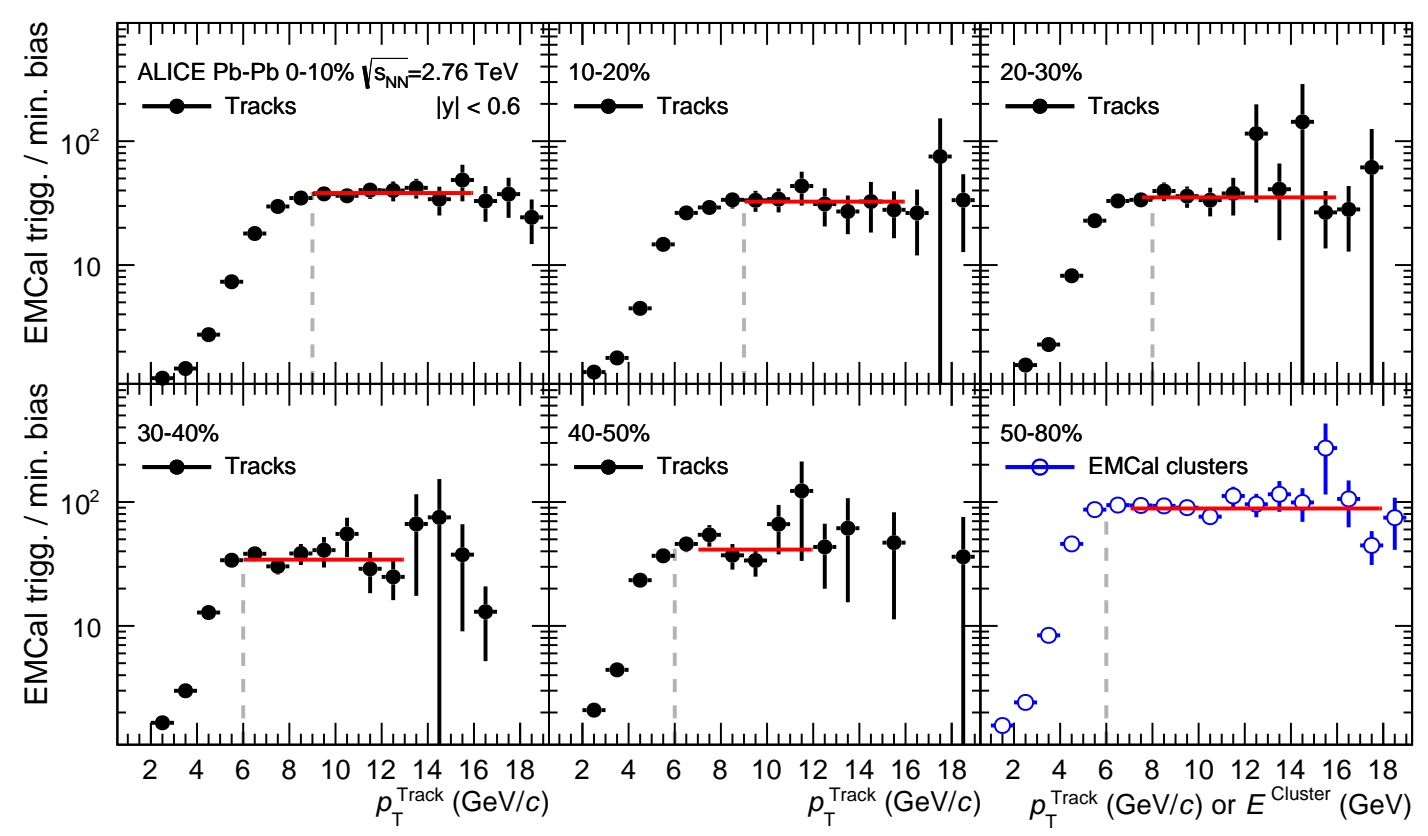

Figure 4: The ratio of inclusive electrons in EMCal triggered events to minimum-bias events as a function of associated track $p_{\mathrm{T}}$ in $\mathrm{Pb}-\mathrm{Pb}$ collisions at $\sqrt{s_{\mathrm{NN}}}=2.76 \mathrm{TeV}$ in centrality bins from $0 \%-10 \%$ and $50 \%-$ $80 \%$. The $p_{\mathrm{T}}$ values which separate the spectra from the minimum-bias trigger and the EMCal trigger, are indicated with black dashed lines.

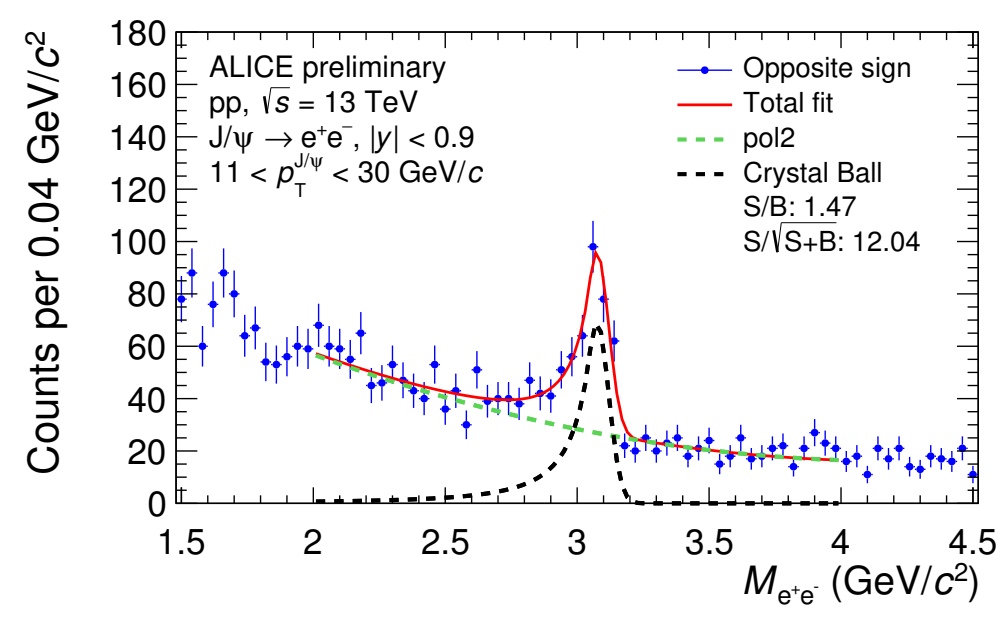

ALI-PREL-130357

Figure 5: Invariant mass spectrum of $e^{+} e^{-}$pairs with $J / \psi$ peak in pp collisions at $\sqrt{s}=13 \mathrm{TeV}$ from EMCal data analysis. 


\section{References}

[1] ALICE collaboration, G. Dellacasa et al., ALICE technical design report of the photon spectrometer (PHOS), CERN-LHCC-99-04.

[2] ALICE EMCAL collaboration, U. Abeysekara et al., ALICE EMCal Physics Performance Report, 1008.0413.

[3] ALICE collaboration, B. Abelev et al., Neutral pion and $\eta$ meson production in proton-proton collisions at $\sqrt{s}=0.9 \mathrm{TeV}$ and $\sqrt{s}=7 \mathrm{TeV}$, Phys. Lett. B717 (2012) 162 [1205.5724].

[4] ALICE collaboration, B. B. Abelev et al., Neutral pion production at midrapidity in $\mathrm{pp}$ and $\mathrm{Pb}-\mathrm{Pb}$ collisions at $\sqrt{s_{\mathrm{NN}}}=2.76 \mathrm{TeV}$, Eur. Phys. J. C74 (2014) 3108 [1405.3794].

[5] ALICE collaboration, S. Acharya et al., $\pi^{0}$ and $\eta$ meson production in proton-proton collisions at $\sqrt{s}=8 \mathrm{TeV}, 1708.08745$.

[6] ALICE collaboration, S. Acharya et al., Neutral pion and $\eta$ meson production in $p$-Pb collisions at $\sqrt{s_{\mathrm{NN}}}=5.02$ TeV, Eur. Phys. J. C78 (2018) 624 [1801.07051].

[7] ALICE collaboration, J. Adam et al., Measurement of the production of high- $p_{\mathrm{T}}$ electrons from heavy-flavour hadron decays in Pb-Pb collisions at $\sqrt{s_{\mathrm{NN}}}=2.76 \mathrm{TeV}$, Phys. Lett. B771 (2017) 467 [1609.07104].

[8] ALICE collaboration, J. Adam et al., Measurement of dijet $k_{T}$ in păASŞPb collisions at $\sqrt{s}_{N N}=5.02$ TeV, Phys. Lett. B746 (2015) 385 [1503.03050].

[9] ALICE collaboration, B. Abelev et al., Measurement of the inclusive differential jet cross section in pp collisions at $\sqrt{s}=2.76$ TeV, Phys. Lett. B722 (2013) 262 [1301.3475].

[10] ALICE collaboration, J. Adam et al., Measurement of jet suppression in central Pb-Pb collisions at $\sqrt{s_{\mathrm{NN}}}=2.76 \mathrm{TeV}$, Phys. Lett. B746 (2015) 1 [1502.01689]. 